\title{
Global Quasi-Real-Time-Services back to Europe: EDRS Global
}

Dr. Harald Hauschildt, Nicolas le Gallou, Silvia Mezzasoma, Hermann Ludwig Moeller, Josep Perdigues Armengol, Dr. Michael Witting (European Space Agency, ESA-ESTEC)

Jörg Herrmann, Cesar Carmona (Airbus CIS, Ottobrunn, Germany) 


\section{European Date Relay System (EDRS) AIRBUS Resa}

EDRS optical data relay services from GEO spacecraft: EDRS-A (90 East) EDRS-C (310 East)

Currently serviced LEO Copernikus satellites:

Sentinel $1 a, b, c, d$ Sentinel $2 a, b, c, d$

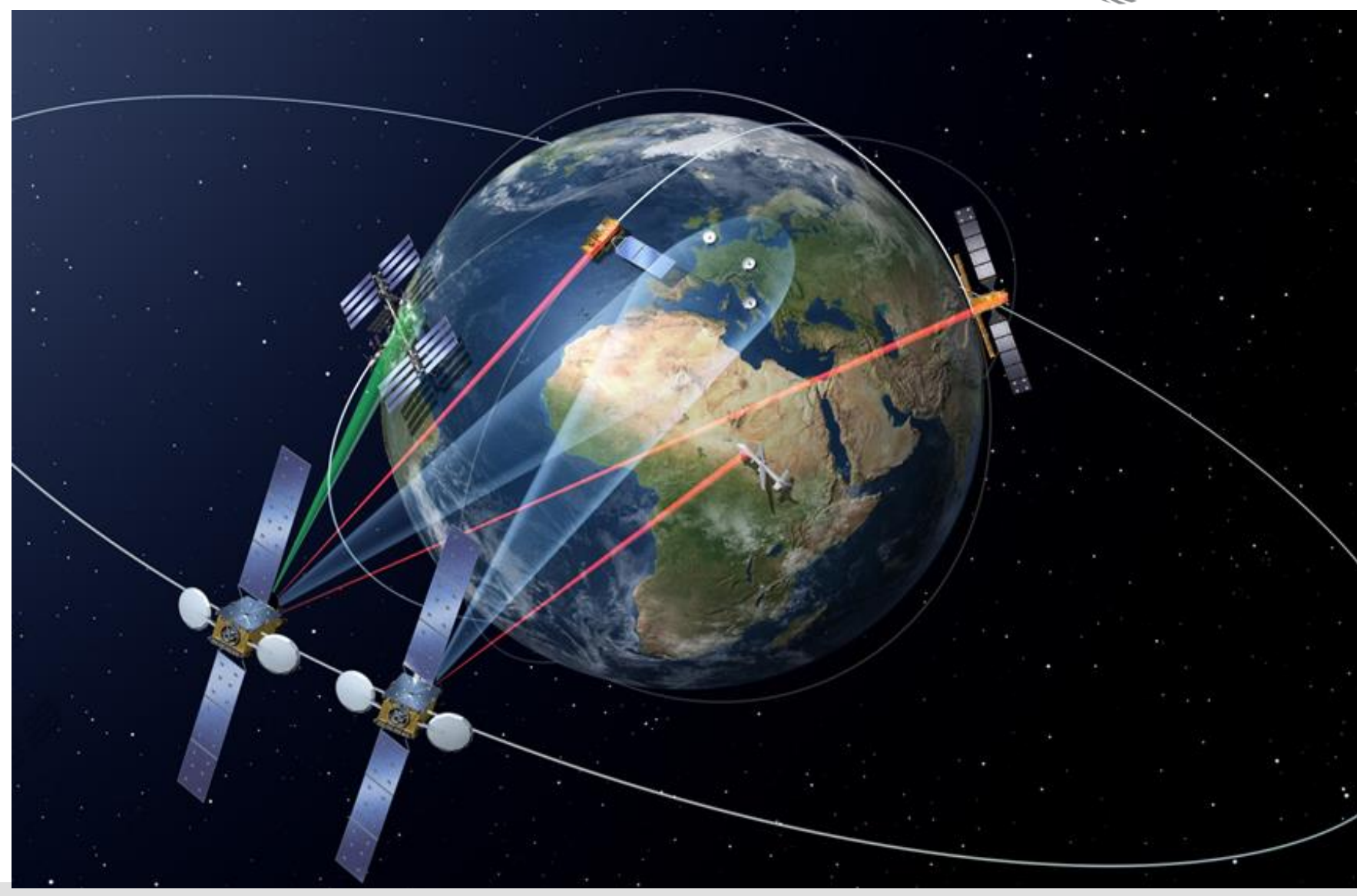




\section{SpaceDataHighway ${ }^{\mathrm{TM}}$}

SpaceDataHighway (SDH) is the Data Relay Service based on the current and future infrastructure of the European Data Relay System (EDRS). It encompasses broadband Laser communication and Ka-band for Inter Satellite Links (ISL).

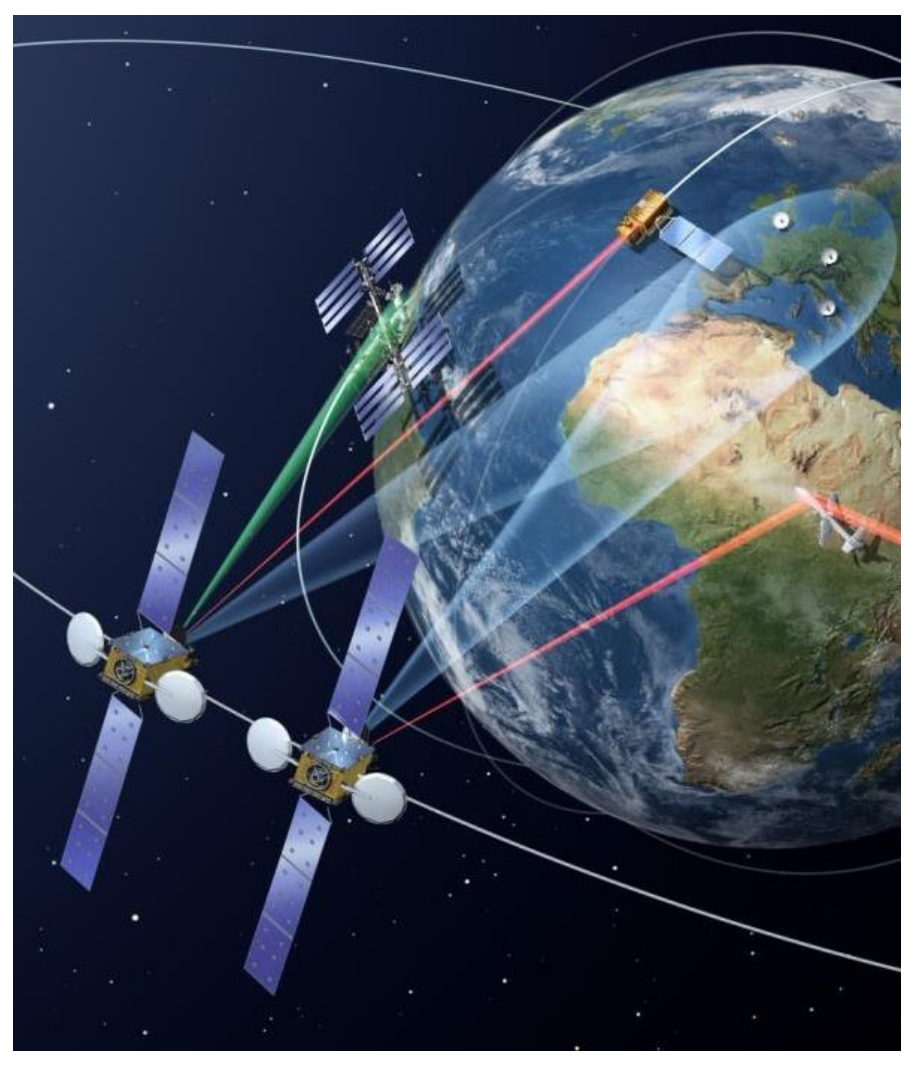




\section{The Service}

AIRBUS Eesa

- has been successfully delivered since November 2016 to the Sentinel-1/2 satellite missions of European Copernicus program

- regular data backhaul from the Sentinel satellites mainly while they are orbiting beyond line of sight of the European ground station network

- is managed from the 24/7 Mission Operations Center (MOC) in Munich

- is initially delivered via the geostationary (GEO) relay node EDRS-A, to be complemented in 2019 by EDRS-C

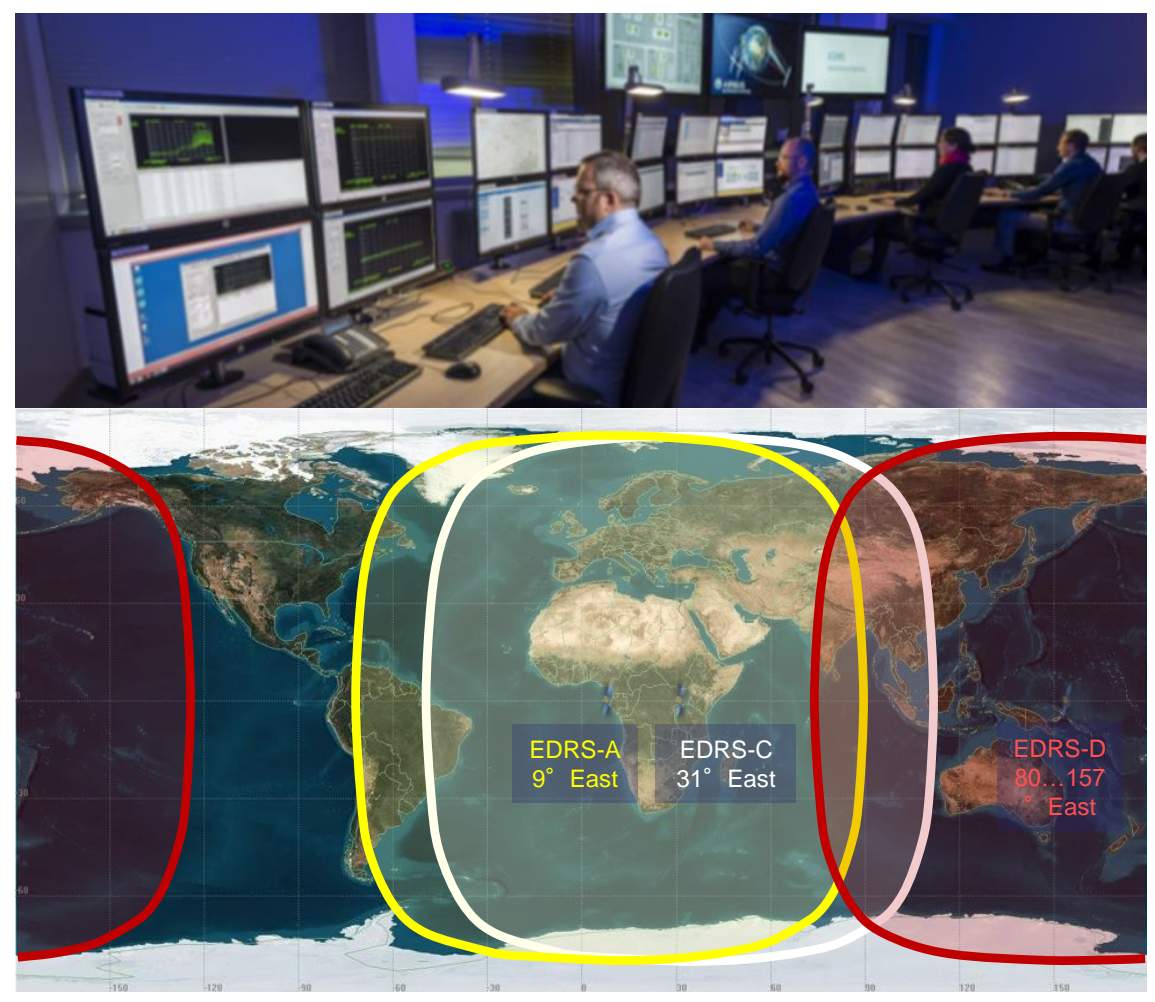




\section{Performance}

- By end 2017 regular data backhaul from four Sentinel satellites

$\checkmark$ increasing data collection capacity by $50 \%$

$\checkmark$ improving data collection flexibility

$\checkmark$ Quasi-Real-Time data transmission capability

- Link times of 10-20 minutes per satellite and orbit

- $\quad$ since Nov 2016

$\checkmark \quad>15000$ successful Laser links

$\checkmark \quad>99 \%$ availability

\section{opernicus}

\section{AIRBUS}

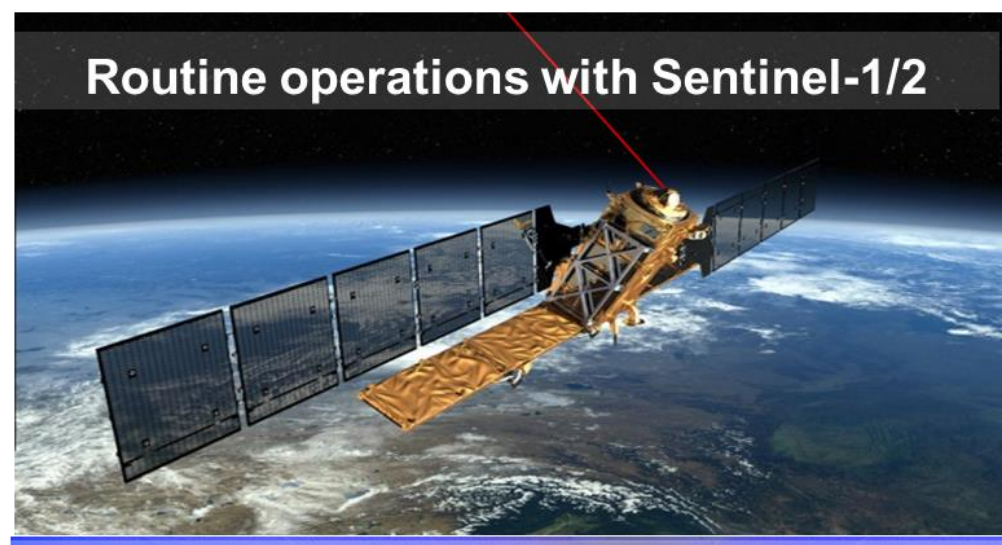

\section{4/7 Mission Operations Center}

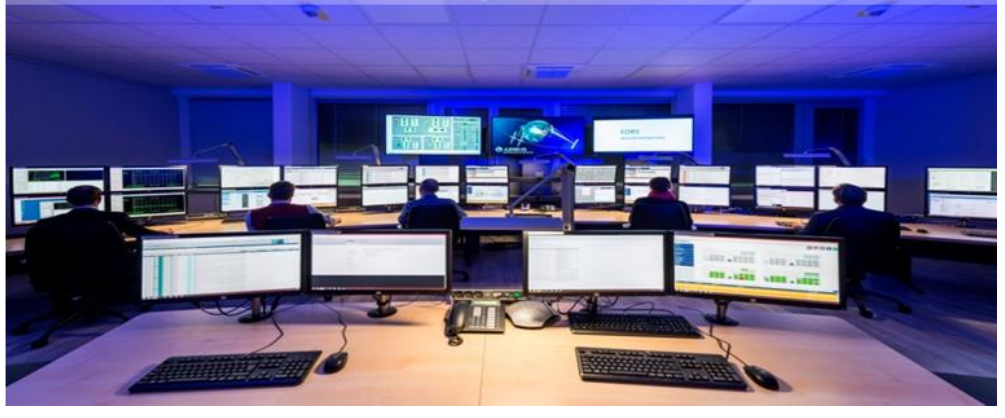

ICSO 2018, Crete | 09/10/2018 | Slide 5 


\section{One Day in a Copernicus routine Service}

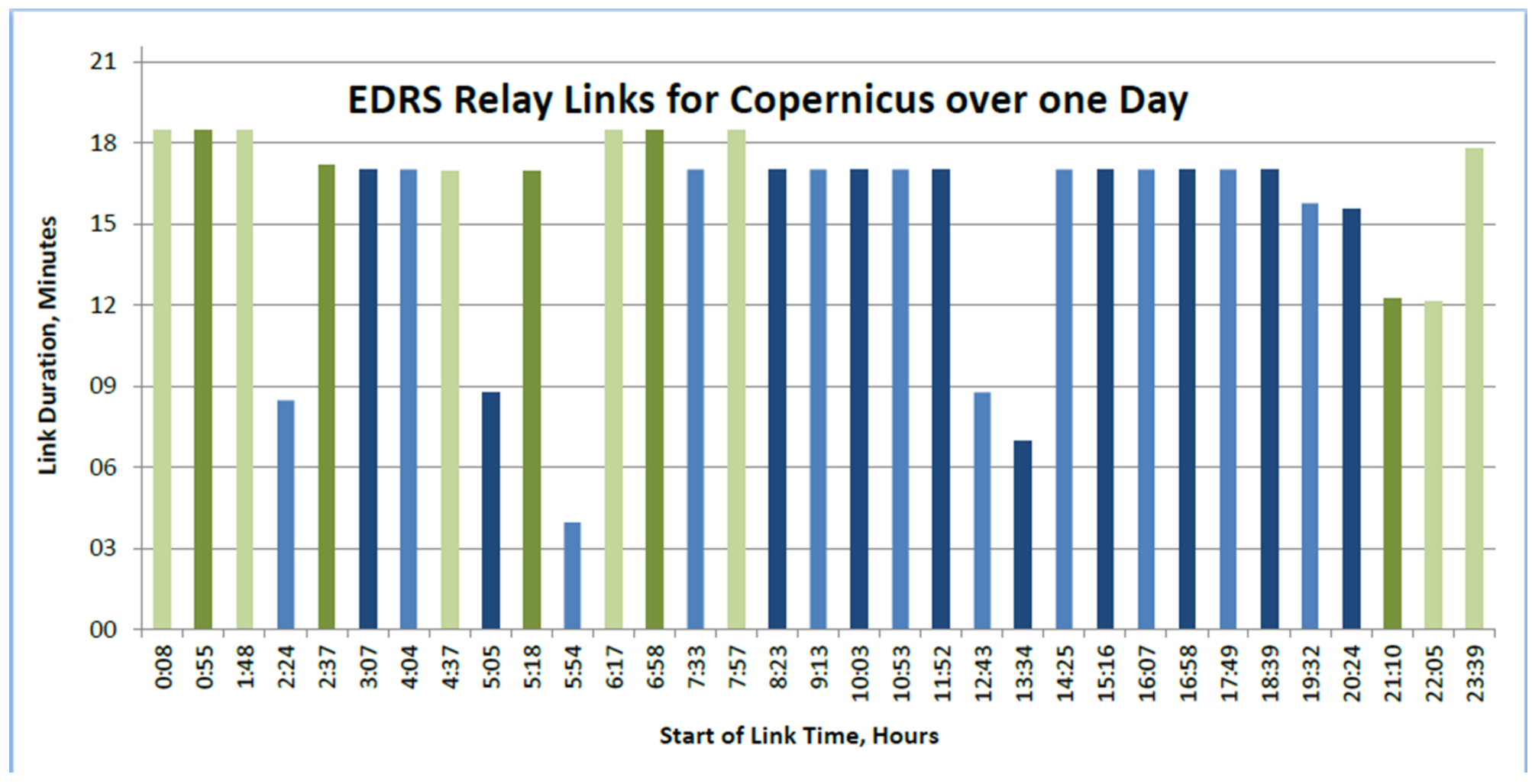

\section{Sentinel}

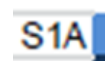

S1B

$\mathrm{S} 2 \mathrm{~A}$

$\mathrm{S} 2 \mathrm{~B}$ 


\section{Commercial Remote Sensing System benefits from SpaceDataHighway}

- Satellites equipped with

$\checkmark$ Laser terminals to transfer data at $1.8 \mathrm{Gbps}$, enabling terabytes/day transmitted securely in quasi-real-time to ground

$\checkmark$ Ka-band terminals allowing last minute tasking beyond ground stations' line-of-sight

- Utilizing SpaceDataHighway for

$\checkmark$ highest system reactivity

$\checkmark$ lowest latency

$\checkmark$ high volume data transfer

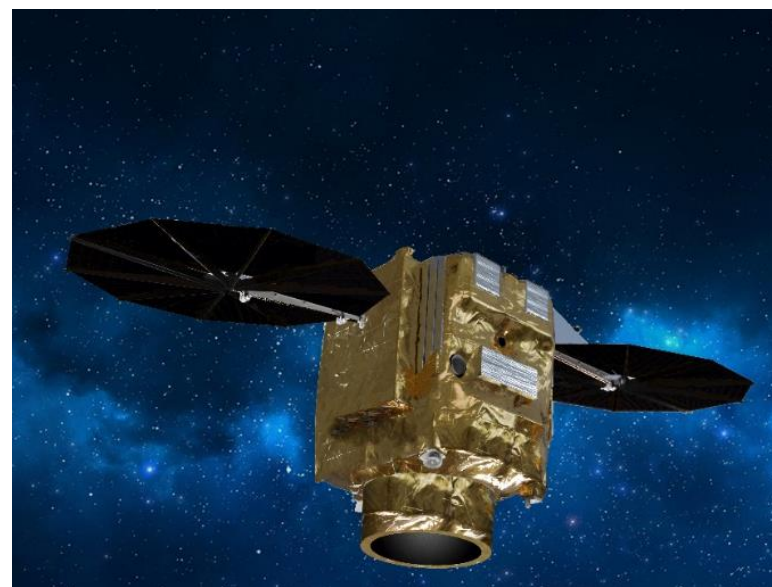

Pléiades Neo

Constellation of four optical VHR satellites Launch in 2020 and 2022

$1 / 2$ million $\mathbf{k m}^{2}$ / day / satellite @ 30 cm

- Delivering increased monitoring capability, optimized operational efficiency (natural disasters, first line response for civil and military applications) 


\section{EDRS-D -Node over Asia/Pacific for Backhauling all your Data}

\section{AIRBUS}

- Service start in $\mathbf{2 0 2 3}$

- Next generation GEO LCTs

$\checkmark \quad$ Interoperability 1064/1550 nm Lasers

$\checkmark$ Bi-directional Laser links with Aircraft

$\checkmark$ Laser Cross Link connecting continents, starting the

"Backbone in Space"

- Airbus examining business opportunities in the EDRS-D project with

Co SKY Perfect JSAT

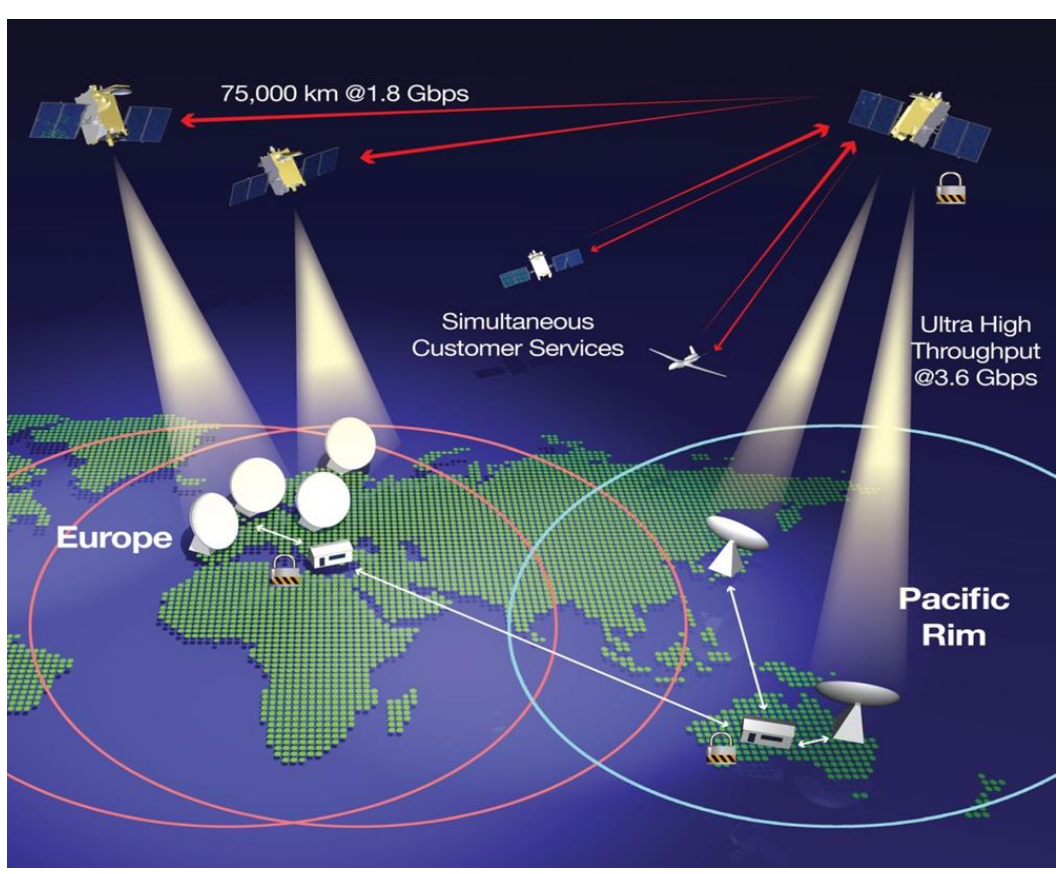

Corporation 
- Next generation data relay node will have an efficient

multi-head Laser communication solution for simultaneous service to multiple users

- Development of improved GEO LCT started to better meet market needs

- New features

$\checkmark$ GEO-GEO cross link

$\checkmark \quad 3.6$ Gbps bandwidth
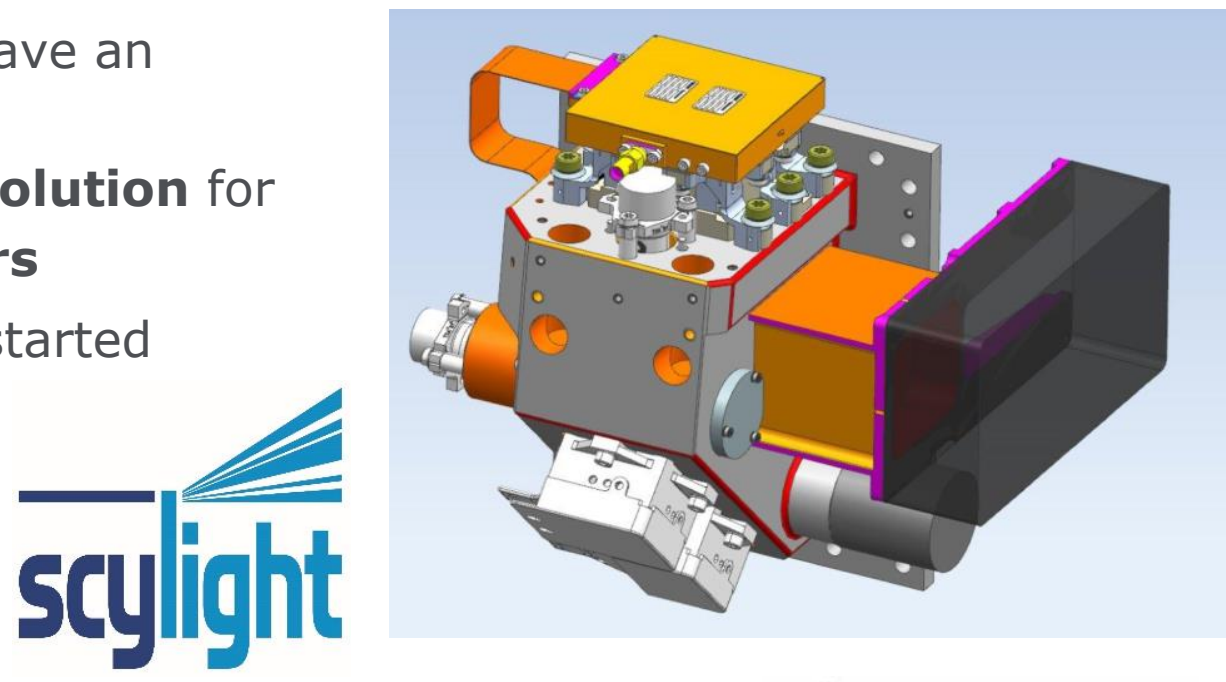

$\checkmark$ Dual Wavelength for interoperability*)

$\checkmark$ Airborne bi-directional

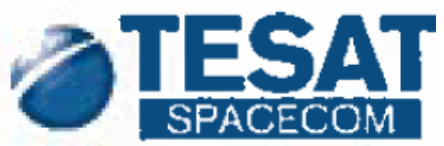

$\checkmark$ Improved operational features

*) in international cooperation 


\section{EDRS Global/SpaceDataHighway for}

Airborne Platforms

\section{AIRBUS}

esa
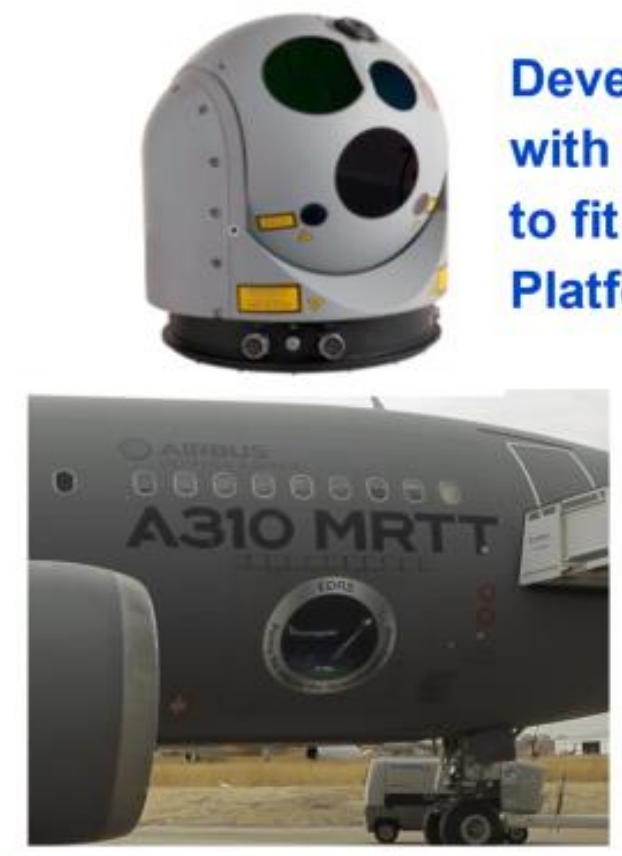

- Bi-directional link for hours/days

- Joint Aerial Layer Network support

- LPI/LPD

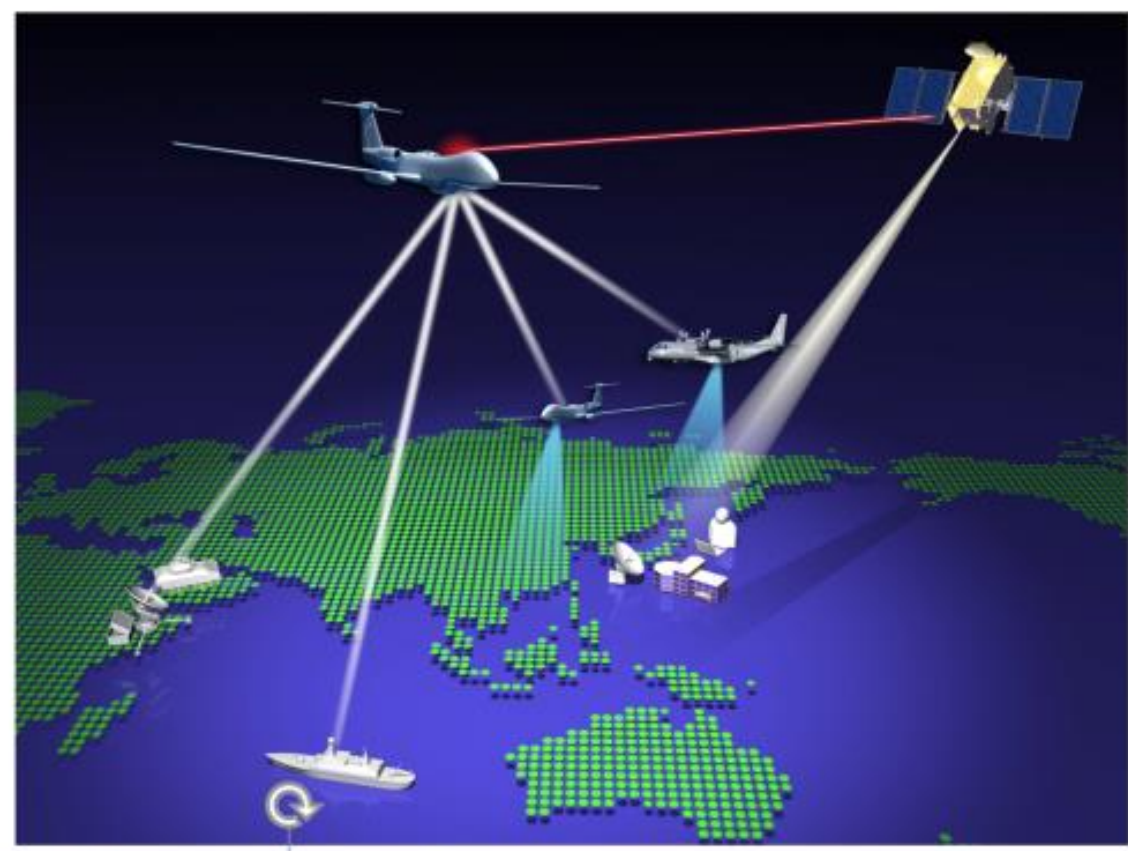

prototyping

demostration 2019

productising

certification operational service 


\section{Airborne and HAPS services}

Permanent Aggregation Node:

- Strategic \& Resilient Laser Comms

- Secured LoS RF communication

- Persistent Airborne Tactical gateway

- Robust, agile network architecture

- LTE Node

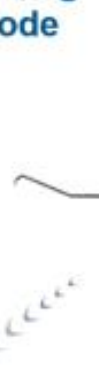

$$
1.8 \mathrm{Gbps}
$$

\section{HAPS}

L16
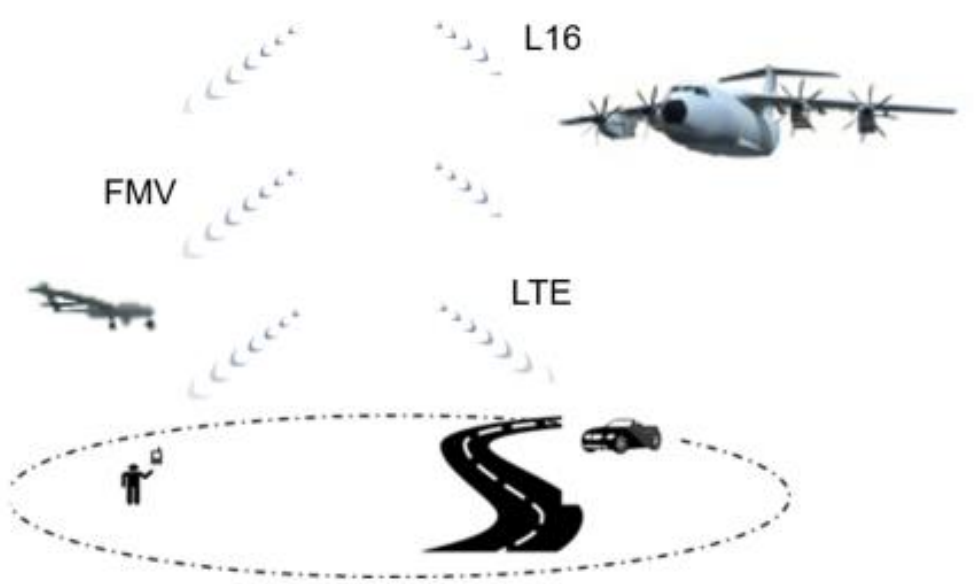

ESA UNCLASSIFIED - For Official Use

\section{AIRBus resa}

\section{Aerial ISR C\&C Node:}

- Strategic \& Resilient Laser Comms

- Secured LoS RF communication

- Airborne Tactical and Strategic Gateway

- Very high bandwidth ISR C\&C Node

$1.8 \mathrm{Gbps}$

backhaul

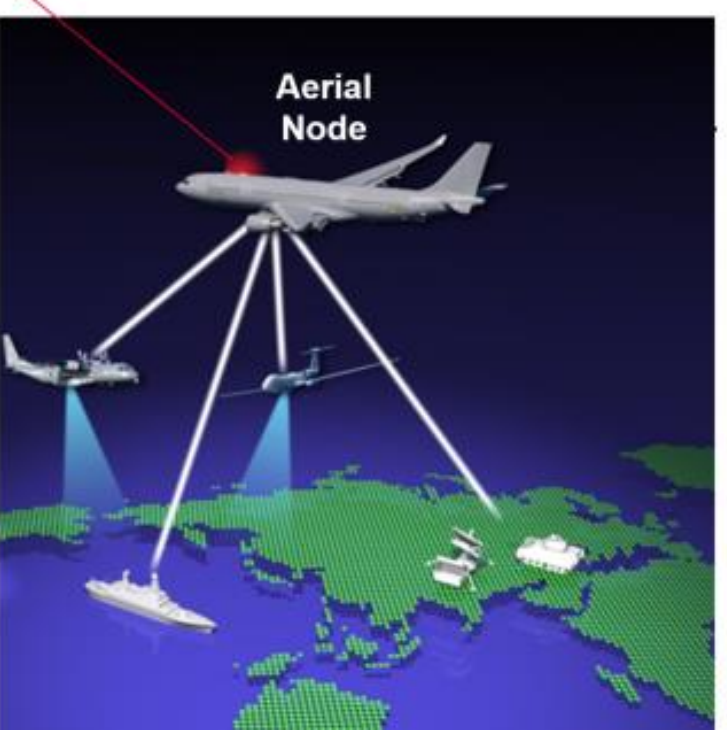

ICSO 2018, Crete | 09/10/2018 | Slide 11 


\section{Conclusions}

- $\quad$ EDRS Global will deploy GLOBAL QRT services for multiple customers

- Copernicus Sentinel Constellation

- Pleiades Neo

- Airborne user platforms

- EDRS Global project is running nominal with a strong industrial coalition

- EDRS Global will be part of ESA CMIN19 proposal to its Member States

\section{Global Quasi-Real-Time-Services by EDRS}

The future is happening right now! 
